$\underline{\text { Preprint typeset in JHEP style. - HYPER VERSION }}$

LPT-Orsay-01-12

hep-ph/0102237

\title{
Nonperturbative corrections to the Drell-Yan transverse momentum distribution
}

\author{
Sofiane Tafat \\ Laboratoire de Physique Théorique,, Université de Paris XI, \\ 91405 Orsay Cedex, France \\ E-mail: Sofiane.Tafat@th.u-psud.fr
}

\begin{abstract}
We study nonperturbative corrections to the transverse momentum distribution of vector bosons in the Drell-Yan process. Factorizing out the Sudakov effects due to soft gluons we express their contribution to the distribution in the form of the vacuum averaged Wilson loop operator. We calculate the nonperturbative contribution to the Sudakov form factor using the expansion of the Wilson loop over vacuum fields supplemented with the expression for nonlocal gauge invariant field strength correlator. Although the Wilson loop is defined in an essentially Minkowski kinematics, the part of the nonperturbative contribution depending on the invariant mass of the produced vector bosons is governed by asymptotics of the correlator at large space-like (Euclidean) separations and therefore can be calculated using conventional nonperturbative methods. Applying the results of lattice calculations we found that the obtained expression for the nonperturbative power corrections is in qualitative agreement with known phenomenological expressions at large transverse momenta and deviate from them at small transverse momenta.
\end{abstract}

\footnotetext{
${ }^{*}$ Unite Mixte de Recherche du CNRS (UMR 8627)
} 


\section{Contents}

1. Introduction 1

2. Factorization of soft gluons 4

3. Perturbative contribution 7

4. IR renormalons in Wilson loops 9

5. Wilson loop in vacuum fields 11

6. Asymptotic behavior of Wilson loop 16

6.1 Small $b^{2}$ behavior $\quad 16$

6.2 Large $b^{2}$ behavior 18

6.3 Ansatz for the nonlocal correlator 18

7. Conclusion 21

\section{Introduction}

Transverse momentum distributions of the vector bosons produced in the Drell-Yan process is one of the classical examples of successful QCD description of the phenomenology of hadron-hadron processes at high energies [1, 2, 3]. Increasing accuracy of the experimental data combined with the state-of-art resummed perturbative calculations allow to test perturbative QCD predictions and, at the same time, estimate nonperturbative corrections to them associated with the confinement effects. It was proposed a long ago to parameterize the latter corrections by introducing additional phenomenological parameters into perturbative formulas [2, 3]. Their values were extracted from the comparison with the experimental data [ [ 1 ]. Despite the fact that the obtained QCD predictions agree well with the experimental data [5] throughout a wide interval of invariant mass of the vector bosons, $Q^{2}$, and their transverse momentum, $q^{2}$, the understanding of nonperturbative effects in the Drell-Yan process is still missing. This should be compared with the situation with hadronization corrections to the deeply inelastic scattering (DIS) and inclusive observables in $e^{+} e^{-}$-annihilation. There, the operator product expansion (OPE) allows to organize the nonperturbative corrections in inverse powers of hard scale $\Lambda_{2 n} / Q^{2 n}$ and identify 
the corresponding nonperturbative scales $\Lambda_{2 n}$ as universal matrix element of composite local operators in QCD like gluon condensate in $e^{+} e^{-}$-annihilation and higher twist operators in DIS. Since the OPE is not applicable to the Drell-Yan production, the physical interpretation of the measured nonperturbative scales remains unclear. Different approaches have been proposed to describe these scales within QCD [6, 7].

In this paper we shall follow the approach developed in [6] and consider nonperturbative corrections the transverse momentum distribution $d^{2} \sigma / d Q^{2} d q^{2}$ in the end-point region describing the production of the vector bosons with large invariant mass $Q^{2}$ and small transverse momentum $q^{2} \ll Q^{2}$. It is well known that both perturbative and nonperturbative corrections are enhanced in this region. Indeed, perturbative QCD analysis shows that the cross section gets large perturbative corrections $\alpha_{s}^{n} / q^{2} \log ^{2 n-1}\left(Q^{2} / q^{2}\right)$. These corrections are associated with multiple soft and collinear gluon emissions and they need to be resummed to all orders in the coupling constant [1]. Applying the Collins-Soper-Sterman resummation formalism one gets [3]

$Q^{2} \frac{d^{2} \sigma}{d Q^{2} d y d q^{2}}=\sigma_{0} \sum_{j} e_{j}^{2} \int \frac{d^{2} b}{(2 \pi)^{2}} \mathrm{e}^{-i \vec{b} \cdot \vec{q}} \mathrm{e}^{-S\left(b^{2}, Q^{2}\right)} C_{j h}\left(x_{1}, Q, b\right) C_{j h^{\prime}}\left(x_{2}, Q, b\right)+Y\left(Q, q, x_{1}, x_{2}\right)$.

Here, $x_{1}=Q / \sqrt{s} \mathrm{e}^{y}$ and $x_{2}=Q / \sqrt{s} \mathrm{e}^{-y}$ are momentum fractions of parton with relative rapidity $y$ and the invariant energy of colliding hadrons $s, \sigma_{0}=\frac{4 \pi \alpha^{2}}{9 s}$ is the Born level partonic cross-section and $C_{j h}$ are given by convolution of partonic distributions $f_{a / h}(x)$ with perturbatively calculable coefficient functions $c_{j a}$

$$
C_{j h}(x, Q, b)=\sum_{a} \int_{x}^{1} \frac{d \xi}{\xi} c_{j a}(x / \xi, b, Q) f_{a / h}\left(\xi, b_{0} / b\right)
$$

with $b_{0}=2 \mathrm{e}^{-\gamma_{E}}$ and $\gamma_{E}$ being the Euler constant. Integration over two-dimensional impact parameter $\vec{b}$ ensures that the total transverse momentum of the vector boson equals $\vec{q}$. In Eq. (1.1), the large perturbative contributions due to soft gluons are factored out into the Sudakov form factor given by

$$
S\left(b^{2}, Q^{2}\right)=\int_{b_{0}^{2} / b^{2}}^{Q^{2}} \frac{d k^{2}}{k^{2}}\left(A\left(\alpha_{s}\left(k^{2}\right)\right) \log \frac{Q^{2}}{k^{2}}+B\left(\alpha_{s}\left(k^{2}\right)\right)\right)
$$

with the coefficient functions $A\left(\alpha_{s}\right)$ and $B\left(\alpha_{s}\right)$ known to two-loop order [2]. The function $Y\left(Q, q, x_{1}, x_{2}\right)$ describes the part of the cross-section (1.1) regular at $q^{2} \ll$ $Q^{2}$. It is obtained by subtracting the logarithmically enhanced part from the fixed order result.

The Sudakov form factor (1.3) resummes large perturbative logarithms $\alpha_{s}^{n} \ln ^{2 n-m}\left(Q^{2} b^{2}\right)$ and it suppresses the large $b^{2}$, or equivalently small $q^{2}$ region of the distribution. Although it is defined for arbitrary $b^{2}$ it was realized that the perturbatively resummed formula (1.1) should be modified at large $b^{2}$. 
Firstly, the expression (1.3) was found by summing of all large logarithms $\log \left(b^{2} Q^{2}\right)$ in the region $Q^{2} \gg 1 / b^{2} \gg \Lambda_{\mathrm{QCD}}^{2}$ where perturbative QCD works. However, since in (1.1) the integration goes over all values of $b^{2}$ the modification of the resummation formula is needed for $1 / b^{2} \sim \Lambda_{\mathrm{QCD}}^{2}$. To this end it was proposed [3, 2] to introduce the scale $Q_{0}$ below which the perturbation QCD fails to replace the impact parameter $b$ by the function

$$
b^{*}=\frac{b}{\left[1+b^{2} Q_{0}^{2}\right]^{1 / 2}}
$$

in the expression for the Sudakov form factor, $S$, and for the distribution function, $C$, in (1.1). Then, at large $b$ thus defined function $b^{*}$ approaches its maximum value $b^{*}=1 / Q_{0}$ and perturbative expressions remain well defined.

Secondly, we expect nonperturbative corrections become equally important at small transverse momentum $q^{2}$, or large $b^{2}$. The latter corrections appear suppressed by powers of both scales, $Q^{2}$ and $q^{2}$. However, for small transverse momentum $q^{2} \ll Q^{2}$ it becomes reasonable to neglect all power corrections on the larger scale, $Q^{2}$, and restrict consideration to nonperturbative effects on the smaller scale $q^{2}$. The standard way to incorporate leading nonperturbative effects into (1.1) amounts to replacing the Sudakov form factor in (1.1) by the following expression [3, 2].

$$
S_{\mathrm{PT}}\left(b^{2}, Q^{2}\right) \rightarrow S_{\mathrm{PT}}\left(\left(b^{*}\right)^{2}, Q^{2}\right)+S_{\mathrm{nonPT}}\left(b^{2}, Q^{2}\right)
$$

with nonperturbative part given by

$$
S_{\mathrm{nonPT}}\left(b^{2}, Q^{2}\right)=\phi(b) \ln \frac{Q}{2 Q_{0}}+\phi_{j / h}\left(b, x_{1}\right)+\phi_{j / h^{\prime}}\left(b, x_{2}\right) .
$$

Here, $\phi$ and $\phi_{j / h}$ are some phenomenological functions whose form should be fitted to the experimental data. The simplest parameterization of the functions looks as follows

$$
\phi(b)=g_{2} b^{2}, \quad \phi_{j / h}\left(b, x_{1}\right)+\phi_{j / h^{\prime}}\left(b, x_{2}\right)=g_{1} b^{2},
$$

The values of the scales $g_{1}$ and $g_{2}$ cannot be calculated in perturbative QCD and one finds them from comparison with the experimental data as [2, [4]

$$
g_{1}=0.24{ }_{-0.07}^{+0.08}(\mathrm{GeV})^{2}, \quad g_{2}=0.34_{-0.08}^{+0.07}(\mathrm{GeV})^{2} \text {. }
$$

There exist other proposals for the parameterization of nonperturbative effects in this process 4 .

In the present paper we study nonperturbative corrections to the resummation formula (1.1) and calculate the power suppressing factor (1.6) using the notion of QCD vacuum condensates. The paper is organized as follows. In Sect. 2 we consider the origin of the Sudakov form factor in the resummation formula (1.1). Using factorization properties of soft gluons we express this form factor as the expectation value of the Wilson loop operator built from soft gluon fields. In Sect. 3 we show 
that the lowest order perturbative calculation of the Wilson loop expectation value reproduces known expression for the form factor (1.3). At the same time, as shown in Sect. 4, to higher orders in perturbation theory this expectation value contains ambiguity associated with the contributions of infrared renormalons having the form of the power correction $\mathcal{O}\left(b^{2} \Lambda^{2}\right)$ [6]. In Sect. 5 we calculate the nonperturbative power corrections to the Wilson loop by taking into account interaction of partons with vacuum gluon fields parameterized by nonlocal QCD condensates. In Sect. 6 we analyze the resummation formula for transverse momentum distribution with nonperturbative effects taking into account and compare it with the phenomenological ansatz (1.5)-(1.7). Sect. 7 contains concluding remarks.

\section{Factorization of soft gluons}

The factorization formula (1.1) is valid up to corrections suppressed by a power of high scale $Q^{2}$ and it takes into account both perturbative and nonperturbative corrections on a smaller scale $q^{2}$ for $q^{2} \ll Q^{2}$. The different factors entering the factorization formula (1.1) take into account contribution from different physical subprocesses hard, collinear and soft - and have the following interpretation. All emissions of quarks and gluons with momenta collinear to incoming hadron momenta $h$ and $h^{\prime}$ are factorized into two distribution functions $f_{a / h}\left(x_{1}, b_{0} / b\right)$ and $f_{b / h^{\prime}}\left(x_{2}, b_{0} / b\right)$. Hard emissions are described by the coefficient functions $c_{j h}$ which, in general, are not universal and depend on the produced vector boson. The soft subprocess describes both the initial state interaction between partons and final state radiation of soft gluons. It is factorized into the Sudakov form factor and will be the central object of our consideration. Its perturbative expression is given by (1.3) and we anticipate that nonperturbative effects will modify it at the level of power corrections $\mathcal{O}\left(\Lambda_{\mathrm{QCD}}^{2 n} b^{2 n}\right)$ which we shall sum over all $n$.

Let us demonstrate that the Sudakov form factor can be calculated in QCD as an expectation value of the Wilson loop operator built from soft gluon fields [8]. Interaction of soft gluons with quarks can be treated using the eikonal approximation. In this way, one can obtain the perturbative expression for the Sudakov form factor by calculating the Feynman diagrams order by order in perturbation theory and replacing quark propagators and quark-gluon vertices by their approximate eikonalized expressions. However, in order to go beyond the perturbation theory one should find an operator definition of the Sudakov form factor that does not refer to the Feynman diagrams and that is given entirely in terms of gluon and quark fields. Such definition exists and it is based on the Wilson line operators defined as

$$
W[C]=P \exp \left(i g \int_{C} d z_{\mu} A^{\mu}(z)\right)
$$

with $A_{\mu}(z)$ being the gauge field describing soft gluons and $C$ being an arbitrary integration path $C$ in the Minkowski space. 
The appearance of the Wilson line operators can be understood as follows [9]. It is well known that in the eikonal approximation the interaction of quark with soft gluons leads to the appearance of the eikonal phase of the quark wave function

$$
\Psi(x) \rightarrow \Phi_{p}[-\infty, x] \Psi(x)
$$

with $\Phi_{p}^{\dagger} \Phi_{p}=1$. The phase $\Phi_{p}[-\infty, x]$ is given by the Wilson line operator (2.1) evaluated along the classical trajectory of quark. For quark with momentum $p_{\mu}$ this trajectory is a straight line that can be parameterize as $z_{\mu}=p_{\mu} s+x_{\mu}$ with $s$ being a proper time

$$
\Phi_{p}[-\infty, x]=P \exp \left(i g \int_{-\infty}^{0} d s p_{\mu} A^{\mu}(p s+x)\right) .
$$

Going over to the momentum representation, it is straightforward to verify that (2.2) and (2.3) reproduce well known expression for the eikonalized amplitude of soft gluon emissions.

In the case of the Drell-Yan process, the Sudakov form factor appears as a contribution of real and virtual soft gluons interacting with incoming quark and antiquark before their annihilation. Applying the eikonal approximation (2.2) we find that both quark and antiquark acquire eikonal phases which are factorized from the amplitude of the process into

$$
U_{\mathrm{DY}}(x)=T\left\{\Phi_{-p_{2}}[-\infty, x] \Phi_{p_{1}}[x,-\infty]\right\},
$$

where $p_{1}^{\mu}$ and $p_{2}^{\mu}$ are momenta of quark and antiquark, respectively, $x_{\mu}$ denotes the annihilation point and $T$ stands for time ordering of gluon field operators. Projecting out the total eikonal phase (2.4) onto all possible final states consisting of an arbitrary number of soft gluons, $N \geq 0$, with the total transverse momenta $(-q)$ we can write the soft gluon contribution to the Drell-Yan cross-section as

$$
\sigma_{\text {eik }}=\sum_{N}\left|\left\langle 0\left|U_{\mathrm{DY}}(0)\right| N\right\rangle\right|^{2} \delta^{(2)}\left(\vec{k}_{N}+\vec{q}\right)=\int \frac{d^{2} b}{(2 \pi)^{2}} \mathrm{e}^{-i \vec{b} \vec{q}} W_{\mathrm{DY}}(b),
$$

where

$$
W_{\mathrm{DY}}(b)=\sum_{N}\left\langle 0\left|U_{\mathrm{DY}}(0)\right| N\right\rangle \mathrm{e}^{-i \vec{b} \vec{k}_{N}}\left\langle N\left|U_{\mathrm{DY}}^{\dagger}(0)\right| 0\right\rangle
$$

and $|N\rangle$ is the final state of $N$ soft gluon with the total momentum $k_{N}^{\mu}$. Using the translation invariance property $U_{\mathrm{DY}}(b)=\mathrm{e}^{i P b} U_{\mathrm{DY}}(0) \mathrm{e}^{-i P b}$ with $P^{\mu}|N\rangle=k_{N}^{\mu}|N\rangle$ being the total momentum operator, one can perform summation over all possible intermediate states to get [6]

$$
W_{\mathrm{DY}}(b)=\left\langle 0\left|U_{\mathrm{DY}}(0) U_{\mathrm{DY}}^{\dagger}(b)\right| 0\right\rangle \equiv\left\langle 0\left|P \exp \left(i g \int_{C_{D Y}} d z_{\mu} A^{\mu}(z)\right)\right| 0\right\rangle .
$$

The integration contour $C_{D Y}$ entering this expression is shown in Fig. 1. It consists of two similar parts having the form of an (infinite) angle which are separated in 
the transverse direction by the impact vector $\vec{b}$. They belong to the plane defined by the quark momenta, $p_{1}^{\mu}$ and $p_{2}^{\mu}$, and correspond to the eikonalized amplitude of the process and its conjugated counterpart. Two cusps located at the space-time points 0 and $b$ define the annihilation points. The cusp angle $\chi$ is equal to the angle between quark momenta $p_{1}$ and $p_{2}$ in Minkowski space

$$
\cosh \chi=\left(p_{1} p_{2}\right) / p^{2}=\frac{Q^{2}}{2 p^{2}}-1,
$$

where $Q^{2}=\left(p_{1}+p_{2}\right)^{2}$ is the invariant mass of produced vector boson and we put $p_{1}^{2}=p_{2}^{2}=p^{2}$ to regularize light-cone singularity that appear for $p^{2} \rightarrow 0$. We find that for $Q^{2} / p^{2} \gg 1$ the cusp angle scales as $\chi=\ln \frac{Q^{2}}{p^{2}}$ and therefore the (kinematical) dependence of the Wilson loop on the cusp angle is translated into $\ln Q^{2}$-dependence of the eikonalized cross-section (2.5).
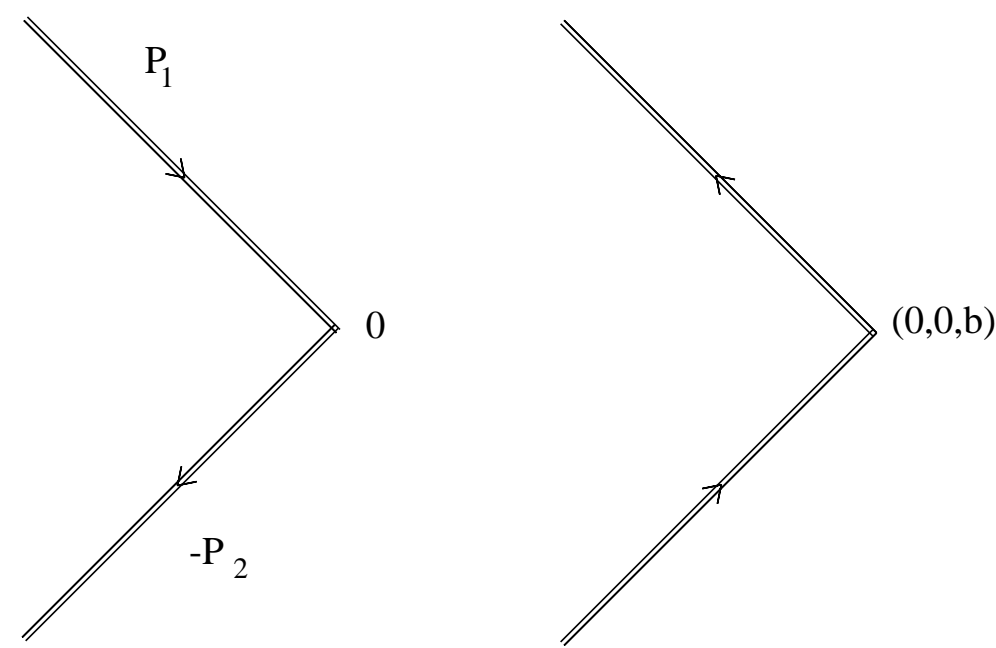

Figure 1: The integration path $C_{\mathrm{DY}}$ defining the eikonal phase (2.7). Straightforward lines correspond to classical trajectories of quark and antiquark with momenta $p_{1}$ and $p_{2}$, respectively.

We would like to notice that the Wilson loop in (2.7) should be averaged with respect to the state describing the spectator partons of both incoming hadrons. In this way one takes into account the possibility for quark and antiquark participating at the partonic subprocess to exchange by soft gluons with constituents of both hadrons before their annihilation. In what follows, we shall neglect such effects for the sake of simplicity and average (2.7) with respect to the vacuum state. There are few reasons why such an approximation is reasonable. First, it is known [9] that all perturbative effects of interaction between hadron constituents and particles participating in short distance partonic subprocess cancel in sum of all Feynman diagram up to corrections suppressed by a power of the hard scale $Q^{2}$. Second, analysis of nonperturbative corrections to the transverse momentum distributions of 
jets produced in $e^{+} e^{-}$-annihilation and deep inelastic scattering indicates [10] that the scale $g_{2}$ parameterizing nonperturbative corrections in Eqs. (1.6) and (1.7) is approximately universal and does not depend on the initial hadronic state.

\section{Perturbative contribution}

Using the expression (2.7) for the Sudakov form factor we may try to take into account both perturbative and nonperturbative effects. The Sudakov form factor depends on $Q^{2}$, lepton pair invariant mass of produced vector boson, and $b^{2}$, impact parameter conjugated to transverse momenta $q$. In the Wilson loop representation (2.7), this dependence follows from the properties of the integration path $C_{D Y}$ in Fig. 1. In particular, the impact parameter $b$ controls the transverse size of the integration contour. As a consequence, putting $\vec{b}=0$ and using unitarity of the eikonal phase one finds that

$$
W_{\mathrm{DY}}(\vec{b}=0)=1
$$

provided that all perturbative (light-cone and short-distance) singularities are appropriately regularized. This suggests to look for an expansion of the Wilson loop expectation value around $\vec{b}=0$. We expect that perturbative expansion will generate $\ln b^{2}$-corrections whereas nonperturbative effects will give rise to corrections in powers of $b^{2}$.

Let us start with perturbative calculation of (2.7) and show that the expression (2.7) reproduces the Sudakov form factor (2) in perturbative QCD at small $b^{2}$. Using the definition (2.7) we get the following expression for the gauge invariant Wilson loop to the lowest order of perturbation theory:

$W^{(1)}=1+\frac{(i g)^{2}}{2} \int_{C_{D Y}} d x^{\mu} \int_{C_{D Y}} d y^{\nu}\left\langle 0\left|A_{\mu}(x) A_{\nu}(y)\right| 0\right\rangle=1-\frac{g^{2}}{2} C_{F} \int_{C_{D Y}} d x^{\mu} \int_{C_{D Y}} d y^{\nu} D^{\mu \nu}(x-y)$

with $C_{F}=\left(N_{c}^{2}-1\right) /\left(2 N_{c}\right)$ and $D_{\mu \nu}(x)$ being a free gluon propagator. To evaluate this integral we have to fix a gauge for a gluon field and, in addition, take care of possible divergencies of the integral. To this end we choose the Feynman gauge and introduce the dimensional regularization with $D=4-2 \varepsilon$ being the dimension of the $x$-space. Substituting the gluon propagator in the Feynman gauge and integrating it twice over the path $C_{\mathrm{DY}}$ we get the following expression for $W^{(1)}$

$$
W^{(1)}=1+g^{2} \mu^{4-D} C_{F} \int \frac{d^{D} k}{(2 \pi)^{D}} 2 \pi \delta_{+}\left(k^{2}\right)\left(\frac{p_{1}}{\left(p_{1} k\right)}-\frac{p_{2}}{\left(p_{2} k\right)}\right)^{2}\left(\mathrm{e}^{i \vec{k} \cdot \vec{b}}-1\right),
$$

where $\mu$ is a dimensionful parameter of the dimensional regularization, $d^{D} k \equiv d k_{+} d k_{-} d^{D-2} \vec{k}$ with $k_{ \pm}$being light-cone components and $\vec{k}$ being $(D-2)$-dimensional transverse momentum of gluon. Integration over $k_{ \pm}$leads to:

$$
W^{(1)}=1+4 \alpha_{s} C_{F}(\chi \operatorname{coth} \chi-1) \mu^{4-D} \int \frac{d^{D-2} \vec{k}}{(2 \pi)^{D-2}} \frac{\mathrm{e}^{i \vec{k} \cdot \vec{b}}-1}{\vec{k}^{2}},
$$


where the cusp angle $\chi$ was defined in (2.8). We notice that this integral does not have infrared divergences at small $\vec{k}^{2}$ but it has ultraviolet divergence coming from large transverse momentum. Dimensional regularization provides a natural cut-off $\mu$ on maximal momenta of soft gluons and, as a consequence, the final expression for $W^{(1)}$ contains a pole in $\varepsilon$

$$
W^{(1)}=1-\frac{\alpha_{s}}{\pi} C_{F}(\chi \operatorname{coth} \chi-1) \frac{\Gamma(1-\varepsilon)}{\varepsilon}\left(\pi \mu^{2} \vec{b}^{2}\right)^{\varepsilon} .
$$

Note that this divergence has nothing to do with conventional UV divergences in perturbative QCD since the residue of a pole in (3.4) depends explicitly on the cusp angle $\chi$ which, by the definition (2.8), is a function of the kinematical invariants of the process. This divergence is introduced by the factorization procedure which separates the contribution of soft gluons from the exact amplitude of the process [11]. Applying the eikonal approximation to the amplitude we correctly describe its IR behavior but change its UV asymptotics. Since by the definition only soft gluons contribute to the Wilson loop expectation value (2.7), we should put an upper bound on the value of the transverse momentum in (3.4). In the dimensional regularization this cut-off is given by $k_{\max }^{2}=\mu^{2}$.

Specific UV divergences which we observed to one-loop order are general feature of Wilson lines. These divergences, the so-called cusp singularities, appear due to the fact that the integration path $C_{\mathrm{DY}}$ entering the definition (2.7) of the Wilson lines contains cusps with angle $\chi$. The renormalization properties of the cusp divergences were studied in detail. It was shown that cusp singularities are renormalized multiplicatively and the corresponding anomalous dimension $\Gamma_{\text {cusp }}\left(\alpha_{s}, \chi\right)$ was found to the lowest orders in perturbative QCD [11].

Subtracting UV pole in the $\overline{M S}$-scheme we get the one-loop renormalized Wilson line as

$$
W^{(1)}=1-\frac{\alpha_{s}}{\pi} C_{F}(\chi \operatorname{coth} \chi-1) \log \frac{\mu^{2} \vec{b}^{2}}{b_{0}^{2}}
$$

where the constant $b_{0}$ was defined in (1.3). Substituting expression (2.8) for the cusp angle $\chi$ we encounter another singularity. Performing calculations of the partonic cross sections in perturbative QCD we neglect quark virtualities by putting $p_{1}^{2}=$ $p_{2}^{2}=0$. In this limit the integration path in Fig. 1 goes along the light-cone and one expects additional singularities to appear. Indeed, using the definition (2.8) we find that the angle $\chi$ is infinite at $p^{2}=0$ and the one-loop expression for $W^{(1)}$ is divergent. As was shown in [1], this singularity originates from soft gluons propagating along the light-cone. The renormalization properties of light-like Wilson lines were studied in [12]. It was found that the renormalized Wilson loop having both light-cone and cusp singularities obeys the following renormalization group equation:

$$
\left(\mu \frac{\partial}{\partial \mu}+\beta(g) \frac{\partial}{\partial g}\right) \frac{\partial \ln W\left(b^{2} \mu^{2}, Q^{2} / \mu^{2}\right)}{\partial \ln Q^{2}}=-2 \Gamma_{\text {cusp }}\left(\alpha_{s}\right)
$$


where $\Gamma_{\text {cusp }}\left(\alpha_{s}\right)$ is the cusp anomalous dimension

$$
\Gamma_{\text {cusp }}\left(\alpha_{s}\right)=\frac{\alpha_{s}}{\pi} C_{F}+\mathcal{O}\left(\alpha_{s}^{2}\right) .
$$

The general solution to this RG equation is given by 8

$$
W\left(b^{2} \mu^{2}, Q^{2} / \mu^{2}\right)=\exp \left(-\int_{b_{0}^{2} / b^{2}}^{\mu^{2}} \frac{d k^{2}}{k^{2}}\left(\Gamma_{\text {cusp }}\left(\alpha_{s}\left(k^{2}\right)\right) \log \frac{Q^{2}}{k^{2}}+\Gamma\left(\alpha_{s}\left(k^{2}\right)\right)\right)\right) W_{0}\left(b^{2}, Q^{2}\right)
$$

where $\Gamma$ is the integration constant and $W_{0}\left(b^{2}, Q^{2}\right)$ is the boundary value of the Wilson loop at the normalization scale $\mu^{2}=b_{0}^{2} / b^{2}$. Since $\mu$ has the meaning of the upper limit on the energy of soft gluons we can put $\mu^{2}=Q^{2}$ in (3.9). Then, the resulting expression for the Wilson loop expectation value (3.9) is given by the product of two factors. One of them resummes all large perturbative logarithms in $b^{2}$ coming from integration over soft gluon transverse momenta above the IR scale $1 / b^{2}$. It coincides with the Sudakov form factor (1.3) provided that the coefficient functions $A\left(\alpha_{s}\right)$ and $B\left(\alpha_{s}\right)$ are given by the anomalous dimensions, $A\left(\alpha_{s}\right)=\Gamma_{\text {cusp }}\left(\alpha_{s}\right)$ and $B\left(\alpha_{s}\right)=\Gamma\left(\alpha_{s}\right)$.

\section{IR renormalons in Wilson loops}

The solution to the evolution equation (3.9) is defined up to the factor $W_{0}\left(b^{2}, Q^{2}\right)$ which gets contribution from soft gluons with transverse momentum below the scale $1 / b^{2}$ and, therefore, cannot be calculated in perturbative QCD. It is this factor that provides nonperturbative contribution to the transverse momentum distribution, Eqs. (1.4) and (1.7), and that we expect to be of the following form

$$
W_{0}\left(b^{2}, Q^{2}\right)=\exp \left(\phi(b) \ln \frac{Q}{2 Q_{0}}+\mathcal{O}\left(\ln ^{0} Q\right)\right) .
$$

Here, $\phi(b)$ is some function of the impact parameter $b^{2}$ which can not be calculated perturbatively. In order to justify (4.1) and to determine the explicit form of the function $\phi(b)$ one has to perform a nonperturbative calculation of the Wilson loop expectation value (2.7). This will be done in the next Section. However, before going through an explicit calculations it becomes instructive to examine the general structure of nonperturbative corrections to $W_{0}\left(b^{2}, Q^{2}\right)$ by calculating the contribution of the IR renormalons to the Wilson loop expectation value (2.7). The IR renormalons describe perturbative contribution to $W\left(b^{2} \mu^{2}, Q^{2} / \mu^{2}\right)$ coming from soft gluon with momenta $k \sim \Lambda_{\mathrm{QCD}}$. The later turns out to be ambiguous at the level of power corrections $\sim \exp \left(-n \beta_{0} / \alpha_{s}\left(1 / b^{2}\right)\right)=\left(b^{2} \Lambda_{\mathrm{QCD}}^{2}\right)^{n}$ with the exponent $n$ uniquely fixed by large order behaviour of the perturbative series. For the physical result (3.9) for

the Wilson loop to be uniquely defined, this ambiguity should be absorbed into the nonperturbative power correction to the boundary condition $W_{0}\left(b^{2}, Q^{2}\right)$. 
To estimate the IR renormalon contribution to the Wilson loop we shall apply the renormalon technique and calculate (2.7) in the so-called single renormalon chain (1-chain) approximation [13]. Applying the Borel transformation we can write the perturbative contribution to the Wilson loop as

$$
W^{(1-\text { chain })}-1=\int_{0}^{\infty} d u \exp \left(-u / \alpha_{s} \beta_{0}\right) B[W](u)
$$

where the Born term was subtracted in the l.h.s. Calculating $W^{(1-\text { chain })}$ we start with the lowest-order expression for the Wilson loop, replace the gluon propagator by the chain of fermionic bubbles and then apply naive nonabelianization to promote the quark contribution to the full one-loop expression for the $\beta$-function. In this way, one finds the following representation for the Borel transform [13]

$$
B[W](u)=-\frac{\sin (\pi u)}{\beta_{0} \pi u \alpha_{s}} \int_{0}^{\infty} d \lambda^{2}\left(\frac{\lambda^{2} \mathrm{e}^{C}}{\mu^{2}}\right)^{-u} \frac{d}{d \lambda^{2}} W^{(1-\text { chain })}\left(\lambda^{2}\right)
$$

where $C$ is a renormalization scheme constant and $C=-5 / 3$ in the $\overline{\mathrm{MS}}-$ scheme. Here, $\lambda^{2}$ is the dispersive parameter which enters into perturbative expression for the Wilson loop in the form of a fictitious gluon mass. This allows us to write $W^{(1-\text { chain) }}\left(\lambda^{2}\right)$ in the form (3.3) with $\delta_{+}\left(k^{2}\right)$ replaced by $\delta_{+}\left(k^{2}-\lambda^{2}\right)$. Integration over longitudinal components $k_{ \pm}$leads to the expression

$$
W^{(1-\text { chain })}\left(\lambda^{2}\right)=1+4 \alpha_{s} C_{F}(\chi \operatorname{coth} \chi-1) \mu^{4-D} \int \frac{d^{D-2} \vec{k}}{(2 \pi)^{D-2}} \frac{\mathrm{e}^{i \vec{k} \cdot \vec{b}}-1}{\vec{k}^{2}+\lambda^{2}},
$$

which should be compared with (3.4). Similar to (3.6), it suffers from collinear singularities as $p^{2} \rightarrow 0$, or $\chi \rightarrow \infty$. To avoid these singularities it suffices to consider the following logarithmic derivative

$$
\frac{d}{d \ln Q^{2}} W^{(1-\text { chain })}\left(\lambda^{2}\right)=C_{F} \frac{\alpha_{s}}{\pi}\left(2\left(\frac{2 \pi b \mu^{2}}{\lambda}\right)^{\varepsilon} K_{\varepsilon}(b \lambda)-\left(\frac{4 \pi \mu^{2}}{\lambda^{2}}\right)^{\varepsilon} \Gamma(\varepsilon)\right)
$$

where $K$ is the Bessel function of the second kind and $D=4-2 \varepsilon$. Then, substituting (4.5) into (4.3) we obtain after some algebra

$$
B\left[\frac{d W}{d \ln Q^{2}}\right](u)=-\frac{C_{F}}{\beta_{0} \pi}\left(\pi b^{2} \mu^{2}\right)^{\varepsilon}\left(b^{2} \mu^{2} / 4 \mathrm{e}^{C}\right)^{u} \frac{\Gamma(1-u-\varepsilon)}{(u+\varepsilon) \Gamma(1+u)} .
$$

This expression for the Borel transform of the Wilson loop in the single chain approximation coincides with the similar expression obtained in [6] using different approach. Finally, substituting (4.6) into (4.2) and assuming that the contribution of a single renormalon chain exponentiates one arrives at

$$
\frac{d \ln W}{d \ln Q^{2}}=-\frac{C_{F}}{\pi \beta_{0}}\left(\pi \mu^{2} \vec{b}^{2}\right)^{\varepsilon} \int_{0}^{\infty} \frac{d u}{u+\varepsilon} \frac{\Gamma(1-u-\varepsilon)}{\Gamma(1+u)}\left(\Lambda_{\mathrm{QCD}}^{2} \vec{b}^{2} / 4 \mathrm{e}^{C}\right)^{u}
$$


Let us analyse the Borel singularities of (4.6) and (4.7). We notice that at $\varepsilon \rightarrow 0$ the r.h.s. of (4.6) contains a pole at $u=0$. It has a UV origin and comes from integration over large transverse momentum in (4.4). As was explained in the previous section, the appearance of this pole is an artefact of the factorization procedure used to separate the contribution of soft gluon. Away from small $u$ we put $\varepsilon=0$ and observe that (4.6) and (4.7) acquires a series of IR renormalon poles located at integer positive $u$. This suggests to divide the integration region in (4.7) into two parts, $0 \leq u \leq 1 / 2$ and $u>1 / 2$, and look for the solutions to (4.7) in the following form

$$
\ln W=\ln W^{(\mathrm{PT})}+\ln W^{(\mathrm{IRR})}
$$

with $W^{(\mathrm{PT})}$ getting a well-defined contribution from small $u$ and $W^{(\mathrm{IRR})}$ taking into account IR renormalon poles.

Integrating over small $u$ in (4.7) we expand the integrand in powers of $u$ to get

$$
\frac{d \ln W^{(\mathrm{PT})}}{d \ln Q^{2}}=\frac{C_{F}}{\pi \beta_{0}} \sum_{n=1}^{\infty}(-1)^{n} \frac{\left(\pi \mu^{2} b^{2} \mathrm{e}^{\gamma_{E}}\right)^{\varepsilon}}{\varepsilon^{n}} \int_{0}^{1 / 2} d u u^{n-1}\left(\Lambda_{Q C D}^{2} b^{2} / b_{0}^{2} \mathrm{e}^{C}\right)^{u}
$$

Subtracting poles in $\overline{\mathrm{MS}}-$ scheme and summing up the series we find

$$
\frac{d \ln W^{(\mathrm{PT})}}{d \ln Q^{2}}=\frac{C_{F}}{\pi \beta_{0}} \log \frac{\ln \left(b_{0}^{2} \mathrm{e}^{C} / \Lambda_{Q C D}^{2} \vec{b}^{2}\right)}{\ln \left(\mu^{2} \mathrm{e}^{C} / \Lambda_{Q C D}^{2}\right)}
$$

where we assumed that $\mu^{2}, 1 / b^{2} \gg \Lambda_{Q C D}^{2}$. It is easy to check that the perturbative contribution to (3.9) satisfies this evolution equation and, as a consequence, $W^{(\mathrm{PT})}$ coincides with the first factor in the r.h.s. of $(3.9)$.

Integrating in (4.7) for $u>1 / 2$ we deform the integration contour around integer positive $u=n$ and estimate the contribution of the IR renormalons to the Wilson loop as

$$
\frac{d \ln W^{(\mathrm{IRR})}}{d \ln Q^{2}}=\sum_{n=1}^{\infty} c_{n}^{\mathrm{IRR}}\left(\Lambda_{Q C D}^{2} \vec{b}^{2} / b_{0}^{2}\right)^{n} \equiv \frac{1}{2} \phi^{\mathrm{IRR}}(b)
$$

with arbitrary coefficients $c_{n}^{\mathrm{IRR}}$ depending on the prescription that one uses integrating the Borel poles and the thus defined $\phi^{\mathrm{IRR}}(b)$ being a function of $b^{2}$ regular at $b=0$. Comparing (4.8) with (3.9) we notice that in order for the Wilson line expectation value $W$ to be well-defined, the IR renormalon ambiguities of $W^{\text {(IRR) }}$ should be absorbed into the definition of the boundary value $W_{0}\left(b^{2}, Q^{2}\right)$. The general form of the IR renormalon contribution can be obtained from (4.10). It matches (4.1) and $\phi^{\mathrm{IRR}}(b)$ provides IR renormalon contribution to the nonperturbative function $\phi(b)$.

\section{Wilson loop in vacuum fields}

As was shown in the previous section, we expect nonperturbative effects to contribute to the power $\mathcal{O}\left(b^{2} \Lambda^{2}\right)$ corrections to the Sudakov form factor. To estimate these 
corrections we shall use the representation of the Sudakov form factor as Wilson loop vacuum expectation value (2.7). The calculation of Wilson loops in QCD has a long history. It is known that for small (Euclidean) size contours we may apply the perturbation theory to calculate the Wilson loop, while for large contours we expect essentially nonperturbative "area law" behavior. There were attempts to justify this behavior using different models of the QCD vacuum [14, 15]. Trying to apply these approaches to the particular Wilson loop shown in Fig. 1 we notice the following important differences. First, the specific feature of the Wilson loop in Fig. 1 is that it has essentially Minkowski light-cone geometry whereas the calculation of a Wilson loop is usually performed in the Euclidian QCD. Second, the integration contour in Fig. 1 is formally of an infinite size, but, at the same times, its definition involves a natural small parameter $\vec{b}^{2}$. At $\vec{b}=0$ the integration path is shrunk into a point and the Wilson loop takes trivial value (2.7). Then, for small $b^{2} \ll 1 / \Lambda_{\mathrm{QCD}}^{2}$ the perturbation theory provides a meaningful approximation to $W(b)$ whereas for $b^{2} \sim 1 / \Lambda_{\mathrm{QCD}}^{2}$ nonperturbative corrections become important.

Following [14, 15], we shall assume that for small $b^{2}$ nonperturbative corrections to $W\left(b^{2}\right)$ come from interaction with vacuum fields. To evaluate the Wilson loop (2.7) we have to fix a gauge and then express the correlator of vacuum gauge fields in terms of gauge invariant quantities. This can be done most easily in the FockSchwinger gauge or fixed point gauge

$$
\left(x-x_{0}\right) \cdot A(x)=0 .
$$

The special feature of this gauge is that the gauge potential can be expressed in terms of field strength tensor $F^{\mu \nu}$ as

$$
A^{\mu}(x)=-\left(x-x_{0}\right)_{\nu} \int_{0}^{1} d \alpha \alpha F^{\mu \nu}\left(x_{0}+\alpha\left(x-x_{0}\right)\right) .
$$

Here, the fixed point $x_{0}$ is a free parameter of the gauge and the $x_{0}$-dependence should cancel in gauge invariant quantities. To simplify the calculations we choose

$$
x_{0}^{\mu}=\xi b^{\mu}
$$

with $\xi$ being an arbitrary gauge parameter. We will demonstrate that the final expression for the Wilson loop does not depend on $\xi$.

Let us introduce the parameterization of the integration path of Fig. 1 as follows: $C_{\mathrm{DY}}=C_{I}+C_{I I}+C_{I I I}+C_{I V}$, where

$$
C_{I}=p_{1} s, \quad C_{I I}=-p_{2} t, \quad C_{I I I}=b-p_{1} t, \quad C_{I V}=b+p_{2} s,
$$

as $-\infty<s \leq 0$ and $0 \leq t<\infty$. Then, using the relation (5.1)-(5.3) we evaluate the integral

$i g \int_{C_{I}} d x \cdot A(x)=i g \int_{-\infty}^{0} d s p_{1}^{\mu} A_{\mu}\left(p_{1} s\right)=i g \xi \int_{-\infty}^{0} d s \int_{0}^{1} d \alpha \alpha p_{1}^{\mu} b^{\nu} F_{\mu \nu}\left(p_{1} s \alpha+b \xi(1-\alpha)\right)$ 
and changing the integration variables $s \rightarrow-s / \alpha$ and $\alpha \rightarrow 1-\alpha / \xi$ we obtain the relation

$$
i g \int_{C_{I}} d x \cdot A(x)=i g \int_{0}^{\infty} d s \int_{0}^{\xi} d \alpha p_{1}^{\mu} b^{\nu} F_{\mu \nu}\left(-p_{1} s+b \alpha\right)
$$

in which $\xi$-dependence enters through the upper integration limit. Similar calculation of the integral along the part $C_{I I}, C_{I I I}$ and $C_{I V}$ leads to the following expressions

$$
\begin{gathered}
i g \int_{C_{I I}} d x \cdot A(x)=-i g \int_{0}^{\infty} d t \int_{0}^{\xi} d \alpha p_{2}^{\mu} b^{\nu} F_{\mu \nu}\left(-p_{2} t+b \alpha\right) \\
i g \int_{C_{I I I}} d x \cdot A(x)=i g \int_{0}^{\infty} d t \int_{\xi}^{1} d \alpha p_{1}^{\mu} b^{\nu} F_{\mu \nu}\left(-p_{1} t+b \alpha\right) \\
i g \int_{C_{I V}} d x \cdot A(x)=-i g \int_{0}^{\infty} d s \int_{\xi}^{1} d \alpha p_{2}^{\mu} b^{\nu} F_{\mu \nu}\left(-p_{2} s+b \alpha\right)
\end{gathered}
$$

which depend separately on $\xi$. Combining together the expressions (5.4) and (5.5), we obtain the following relation

$$
i g \int_{C} d x \cdot A(x)=i g \int_{0}^{\infty} d s \int_{0}^{1} d \alpha b^{\nu}\left(p_{1}^{\mu} F_{\mu \nu}\left(-p_{1} s+b \alpha\right)-p_{2}^{\mu} F_{\mu \nu}\left(-p_{2} s+b \alpha\right)\right)
$$

in which the dependence of the gauge parameter $\xi$ disappears.

Finally, substituting (5.6) into (3.2) we find that to the lowest order in a gauge field expansion the vacuum averaged Wilson loop is given by

$$
W=1-\frac{1}{4 N}\left\langle 0\left|g^{2}\left[\int_{0}^{\infty} d s \int_{0}^{1} d \alpha b^{\nu}\left(p_{1}^{\mu} F_{\mu \nu}^{a}\left(-p_{1} s+b \alpha\right)-p_{2}^{\mu} F_{\mu \nu}^{a}\left(-p_{2} s+b \alpha\right)\right)\right]^{2}\right| 0\right\rangle
$$

Although this expression is $\xi$-independent, it is not gauge invariant. To restore the gauge invariance, two field strength tensors should be connected by a Wilson line to form a nonlocal correlator $F_{\mu \rho}^{a}(x)\left[P \exp \left(i g \int_{y}^{x} d z \cdot A^{c}(z) t^{c}\right)\right]_{a b} F_{\nu \lambda}^{b}(y)$ with the group generators $t^{a}$ defined in the adjoint representation of the $S U(N)$ group. Here, the integration path can be an arbitrary and we choose a straightforward line for simplicity. Any other choice leads to the expression for the Wilson loop which is different from (5.7) by terms containing higher powers of gauge fields. Thus defined gauge invariant field strength correlator depends on the difference $(x-y)$ and it can be decomposed onto two Lorentz tensors [14, [16]:

$$
\begin{aligned}
\left\langle 0\left|g^{2} F_{\mu \rho}^{a}(x)\left[P \mathrm{e}^{i g \int_{0}^{x} d z \cdot A(z)}\right]_{a b} F_{\nu \lambda}^{b}(0)\right| 0\right\rangle & =\left(g_{\mu \nu} g_{\rho \lambda}-g_{\mu \lambda} g_{\nu \rho}\right) \Phi_{1}\left(-x^{2}+i \epsilon\right) \\
& +\left(g_{\rho \alpha} \partial_{\mu}-g_{\mu \alpha} \partial_{\rho}\right)\left(g_{\lambda \alpha} \partial_{\nu}-g_{\nu \alpha} \partial_{\lambda}\right) \Phi_{2}\left(-x^{2}+i \epsilon\right)
\end{aligned}
$$

Here, $\Phi_{1}$ and $\Phi_{2}$ are gauge invariant functions that receive both perturbative and nonperturbative corrections. In the special case of abelian gauge group the Bianchi identity $\epsilon^{\mu \nu \rho \lambda} \partial_{\mu} F_{\nu \rho}=0$ implies that $\Phi_{1}\left(-x^{2}\right)=0$. Although it is not the case for 
QCD, this property leads to vanishing the lowest order perturbative QCD correction to $\Phi_{1}$. It is convenient to introduce the following representation

$$
\begin{aligned}
\Phi_{1}\left(-x^{2}+i \epsilon\right) & =-i \int_{0}^{\infty} d \sigma \mathrm{e}^{-i \sigma x^{2}} \tilde{\Phi}_{1}(i \sigma), \\
\frac{d \Phi_{2}\left(-x^{2}+i \epsilon\right)}{d x^{2}} & =-i \int_{0}^{\infty} d \sigma \mathrm{e}^{-i \sigma x^{2}} \tilde{\Phi}_{2}(i \sigma),
\end{aligned}
$$

where we took into account that eq. (5.8) defines the function $\Phi_{2}$ up to an arbitrary constant and it is the derivative of this function that should enter into the final expressions for the Wilson loop. The correlator (5.8) receives both perturbative and nonperturbative corrections. To the lowest order of perturbation theory we use the definition (5.8) to calculate the functions $\tilde{\Phi}_{1}(\sigma)$ and $\tilde{\Phi}_{2}(\sigma)$ as

$$
\tilde{\Phi}_{1}^{(\mathrm{PT})}(\sigma)=0, \quad \tilde{\Phi}_{2}^{(\mathrm{PT})}(\sigma)=-\frac{g^{2}}{4 \pi^{D / 2}}\left(N^{2}-1\right) \mu^{4-D} \sigma^{D / 2-1} .
$$

Let us substitute the correlator (5.8) into the expression (5.7) for the Wilson loop. Contracting the Lorentz indices, using the identities $\left(p_{1} \cdot b\right)=\left(p_{2} \cdot b\right)=0$ and performing integration over $s$ in (5.7) one arrives at

$$
\left(p_{1} p_{2}\right) \int_{0}^{1} d s d t \mathrm{e}^{-i \sigma\left(p_{1} s+p_{2} t\right)^{2}}=\frac{1}{2 i \sigma} \chi \operatorname{coth} \chi
$$

where $\chi$ is the angle between quark and antiquark momenta defined in (2.8). Here, we put quark momenta off-shell, $p_{1}^{2}=p_{2}^{2} \neq 0$, in order to avoid light-cone singularities. The remaining integral in (5.7) is given by

$$
f\left(i \sigma b^{2}\right) \equiv i \sigma b^{2} \int_{0}^{1} d \alpha \int_{0}^{1} d \beta \mathrm{e}^{-i \sigma b^{2}(\alpha-\beta)^{2}}
$$

and it can be expressed in terms of the error function $\operatorname{erf}(x)=\frac{2}{\sqrt{\pi}} \int_{0}^{x} d t \mathrm{e}^{-t^{2}}$ as

$$
f(x)=2 x \int_{0}^{1} d \alpha(1-\alpha) \mathrm{e}^{-x \alpha^{2}}=\mathrm{e}^{-x}-1+\sqrt{\pi x} \operatorname{erf}(\sqrt{x})
$$

for an arbitrary $x$. Finally, we obtain the following expression for the Wilson loop (5.7)

$$
W=1-\frac{i}{4 N}(\chi \operatorname{coth} \chi-1) \int_{0}^{\infty} \frac{d \sigma}{\sigma^{2}}\left[\tilde{\Phi}_{1}(i \sigma) f\left(i \sigma \vec{b}^{2}\right)-2 \tilde{\Phi}_{2}(i \sigma)\left(1-\mathrm{e}^{-i \sigma \vec{b}^{2}}\right)\right],
$$

where the functions $\tilde{\Phi}_{1}$ and $\tilde{\Phi}_{2}$ parameterize the correlator (5.8). The following comments are in order.

The dependence of the Wilson loop on the invariant mass $Q^{2}$ and impact parameter $b^{2}$ is factorized in (5.14) into two different factors. The $Q^{2}$-dependence is given by a "cusp factor" $(\chi \operatorname{coth} \chi-1)$ which coincides with an analogous factor in the perturbative expression (3.4). At the same time, to evaluate the $b^{2}$-dependence of 
$W$ we need to know nonperturbative functions $\tilde{\Phi}_{1}$ and $\tilde{\Phi}_{2}$. Notice that in the original expression for the Wilson loop (5.7) the nonlocal gluon correlator was integrated over both space-time and like-like separations $x^{2}$ corresponding to the distance between different points on the contour $C_{\mathrm{DY}}$, whereas the final expression (5.14) contains the integral over parameter $\sigma$ and depends on the space-like vector $b$. This suggests to perform the Wick rotation: $\sigma \rightarrow-i \sigma$ and rewrite (5.14) in the limit $Q^{2} \rightarrow \infty$ as

$$
\frac{d \ln W}{d \ln Q^{2}}=-\frac{1}{4 N} \int_{0}^{\infty} \frac{d \sigma}{\sigma^{2}}\left[\tilde{\Phi}_{1}(\sigma) f\left(\sigma \vec{b}^{2}\right)-2 \tilde{\Phi}_{2}(\sigma)\left(1-\mathrm{e}^{-\sigma \vec{b}^{2}}\right)\right] .
$$

The advantage of this representation with respect to (5.14) is that the light-cone singularities cancel out in the r.h.s. of (5.15) and therefore it has a well defined limit as quark momenta go on-shell, $p_{1}^{2}=p_{2}^{2}=0$.

The above mentioned properties of the Wilson loop (5.14) and (5.15) can be understood as follows. As was shown in the previous section, leading nonperturbative $\sim b^{2}$ corrections to the Wilson loop come from the region of small transverse momenta whereas collinear $\operatorname{logs} \sim \ln Q^{2} / p^{2}$ appear from integration over small angles between gluon momentum and one of the quark momenta $p_{1}$ and $p_{2}$. Since integration over small angles and small transverse momenta can be performed independently, the $Q^{2}$-dependence of perturbative and nonperturbative expressions, Eqs. (3.4) and (5.14), respectively, coincide. The remaining integration over transverse momenta of gluon is translated into $\sigma$-integration in (5.14) with nonperturbative corrections absorbed into the functions $\widetilde{\Phi}_{1}(\sigma)$ and $\widetilde{\Phi}_{2}(\sigma)$.

Expression (3.2) defines first two terms in the expansion of the Wilson loop over vacuum gauge field. Natural question arises about the contribution of the remaining terms in this expansion. It can be estimated by applying the nonabelian exponentiation theorem [17] which allows to distinguish higher order terms according to their color structure. It leads to the following general expression for the Wilson loop

$$
W=\exp \left(w_{1}+w_{2}+\ldots\right)
$$

where $w_{n}$ is the total contribution to the Wilson loop $W$ with "maximally nonabelian" color factors, $w_{n} \sim C_{F} N_{c}^{n-1}$, the so-called "webs". Expanding this expression one can express the contribution to the Wilson loop with arbitrary color factor in terms of maximally nonabelian webs which can be defined without reference to a perturbative expansion. Comparing (5.16) with (5.14) we find that obtained expression for $W$ provides a lowest order contribution to the web $w_{1}$. Then, applying the nonabelian exponentiation theorem we may exponentiate the lowest order expression (5.14) and write the Wilson loop in the form (5.16) modulo corrections coming from higher webs. According to (3.4), $w_{1}$ has the following asymptotics in large $Q^{2}$ limit: $w_{1}=\mathcal{O}\left(\ln Q^{2}\right)$. It can be shown following [11], that this is a general property of the webs corresponding to the Wilson loop shown in Fig. 1, $w_{n} \sim \ln Q^{2}$. Therefore, being combined together in the exponent of (5.16), higher order webs $w_{n}$ renormalize 
the coefficient in front of collinear logarithm $\sim \ln Q^{2}$ in lowest web contribution $w_{1}$. This property implies that the derivative $d \ln W / d \ln Q^{2}$ does not contain collinear logs and, therefore, it is $Q^{2}$-independent as $Q^{2} \rightarrow \infty$ in agreement with (5.15).

Substituting (5.10) into (5.15) and subtracting pole in the $\bar{M} S$-scheme, one finds that perturbative contribution to the Wilson loop satisfies the following evolution equation

$$
\frac{d \ln W^{(\mathrm{PT})}\left(Q^{2} b^{2} / b_{0}^{2}\right)}{d \ln Q^{2}}=-\int_{b_{0}^{2} / b^{2}}^{Q^{2}} \frac{d k_{\perp}^{2}}{k_{\perp}^{2}} \Gamma_{\text {cusp }}\left(\alpha_{s}\left(k_{\perp}^{2}\right)\right)
$$

where $\Gamma_{\text {cusp }}$ given by (3.8). The higher order webs provide a higher order perturbative contribution to the anomalous dimension $\Gamma_{\text {cusp }}$. Integrating (5.17) one reproduces perturbative contribution to (3.9). Applying the nonabelian exponentiation theorem to calculate nonperturbative contribution to the Wilson loop we arrive at the evolution equation (5.15) which is valid up to higher web contribution. The evolution equation (5.15) allows to calculate the coefficient in front of $\ln Q^{2}$ but it does not fix the corresponding scale $Q_{0}^{2}$

$$
W_{0}\left(b^{2}, Q^{2}\right)=\exp \left(-\frac{1}{4 N} \log \frac{Q^{2}}{Q_{0}^{2}} \int_{0}^{\infty} \frac{d \sigma}{\sigma^{2}}\left[\tilde{\Phi}_{1}(\sigma) f\left(\sigma \vec{b}^{2}\right)-2 \tilde{\Phi}_{2}(\sigma)\left(1-\mathrm{e}^{-\sigma \vec{b}^{2}}\right)\right]\right) .
$$

This expression was first obtained in [18. It is valid up to higher order webs contribution and it is assumed that perturbative contribution is subtracted from the functions $\Phi_{1}$ and $\Phi_{2}$. One finds similar expression for the Wilson loop using the stochastic model for the QCD vacuum [14]. There, it corresponds to the neglecting the contributions of higher cumulants of gauge strength fields.

\section{Asymptotic behavior of Wilson loop}

Let us consider the properties of nonperturbative contribution to the Wilson loop (5.18). As follows from (5.18), the $b^{2}$-asymptotics of the exponent is determined by the properties of the function $f\left(\sigma b^{2}\right)$ defined in (5.13).

\subsection{Small $b^{2}$ behavior}

Using the definition (5.9), we find that $f(x)$ takes positive values for $x>0$ and for small $x$ it has the following asymptotics

$$
f(x)=x-\frac{x^{2}}{6}+\frac{x^{3}}{30}-\frac{x^{4}}{168}+\ldots, \quad \text { for } x \ll 1 .
$$

Thus, the exponent of the Wilson loop (5.18) admits the following expansion in powers of $b^{2}$

$$
W_{0}=\exp \left(-\ln \frac{Q^{2}}{Q_{0}^{2}}\left[c_{1} b^{2}+c_{2} b^{4}+c_{3} b^{6}+\mathcal{O}\left(b^{8}\right)\right]\right)
$$


where the coefficients $c_{n}$ are defined as moments of the functions $\tilde{\Phi}_{1}(\sigma)$ and $\tilde{\Phi}_{2}(\sigma)$ :

$$
\begin{aligned}
& c_{1}=\frac{1}{4 N} \int_{0}^{\infty} \frac{d \sigma}{\sigma}\left(\tilde{\Phi}_{1}(\sigma)-2 \tilde{\Phi}_{2}(\sigma)\right) \\
& c_{2}=-\frac{1}{24 N} \int_{0}^{\infty} d \sigma\left(\tilde{\Phi}_{1}(\sigma)-6 \tilde{\Phi}_{2}(\sigma)\right) \\
& c_{3}=\frac{1}{120 N} \int_{0}^{\infty} d \sigma \sigma\left(\tilde{\Phi}_{1}(\sigma)-10 \tilde{\Phi}_{2}(\sigma)\right)
\end{aligned}
$$

The expansion (6.2) is in agreement with analysis of the IR renormalon ambiguities of perturbative series for $W$ performed in Sect. 4 . Dimensionful parameters $c_{n}$ are related to the nonlocal correlator (5.8). The explicit form of these relations can be found from the definition $(5.9)$ as

$$
\begin{aligned}
& c_{1}=\frac{1}{4 N} \int_{0}^{\infty} d \vec{x}^{2}\left[\Phi_{1}\left(\vec{x}^{2}\right)+2 \Phi_{2}^{\prime}\left(\vec{x}^{2}\right)\right] \\
& c_{2}=-\frac{1}{24 N}\left[\Phi_{1}(0)+6 \Phi_{2}^{\prime}(0)\right] \\
& c_{3}=-\frac{1}{120 N}\left[\Phi_{1}^{\prime}(0)+10 \Phi_{2}^{\prime \prime}(0)\right],
\end{aligned}
$$

where prime denotes differentiation with respect to $x^{2}$. These equations imply that all dimensionful coefficients $c_{n}$ except of $c_{1}$ are related to the short distance asymptotics of the nonlocal correlator (5.8). Expanding the l.h.s. of (5.8) in powers of $x^{2}$ one can calculate the first few terms of the small $x^{2}$ expansion of the functions $\Phi_{1}\left(-x^{2}\right)$ and $\Phi_{2}\left(-x^{2}\right)$ in terms of local vacuum condensates as

$$
\begin{aligned}
\Phi_{1}\left(-x^{2}\right) & =\frac{\left\langle g^{2} F^{2}\right\rangle}{12}+x^{2} \frac{\left\langle g^{3} f F^{3}\right\rangle}{48}+\mathcal{O}\left(x^{4}\right), \\
\frac{d \Phi_{2}\left(-x^{2}\right)}{d x^{2}} & =-x^{2} \frac{\left\langle g^{4} j^{2}\right\rangle}{288}+\mathcal{O}\left(x^{4}\right)
\end{aligned}
$$

where $\left\langle g^{3} f F^{3}\right\rangle \equiv\left\langle g^{3} f^{a b c} F_{\mu \nu}^{a} F_{\nu \rho}^{b} F_{\rho \mu}^{c}\right\rangle,\left\langle g^{4} j^{2}\right\rangle \equiv\left\langle g^{4} j_{\mu}^{a} j_{\mu}^{a}\right\rangle$ and $j_{\mu}^{a}$ is a quark current. Using these relations we evaluate the coefficients $c_{2}$ and $c_{3}$ as

$$
c_{2}=-\frac{\left\langle g^{2} F^{2}\right\rangle}{288 N}, \quad c_{3}=\frac{1}{1152 N}\left(\frac{\left\langle g^{3} f F^{3}\right\rangle}{5}-\frac{\left\langle g^{4} j^{2}\right\rangle}{3}\right)
$$

We would like to stress that, in general, the scale $c_{1}$ is not related to local vacuum condensates. $^{2}$ To find its value one needs to know the behavior of the correlator (5.8) at large (Euclidean) distances.

\footnotetext{
${ }^{2}$ This is in agreement with the fact that there are no local gauge invariant operators in QCD of the dimension 2 .
} 


\subsection{Large $b^{2}$ behavior}

The expansion (6.1) and (6.2) has a finite radius of convergence and it is not applicable at large $x$, or equivalently large impact vectors $\vec{b}^{2}$. Using the definition (5.9) we find the asymptotic behaviour of the function $f(x)$ at large $x$ as

$$
f(x)=\sqrt{\pi x}-1+\mathrm{e}^{-x}\left(\frac{1}{2 x}-\frac{3}{4 x^{2}}+\ldots\right), \quad \text { for } x \gg 1 .
$$

Its substitution into (5.18) yields

$$
W_{0}\left(b^{2}, Q^{2}\right)=\exp \left(-\ln \frac{Q^{2}}{Q_{0}^{2}}\left[c_{0} \sqrt{\vec{b}^{2}}+\mathcal{O}\left(b^{0}\right)\right]\right),
$$

where the dimension 1 coefficient $c_{0}$ is defined as

$$
c_{0}=\frac{\sqrt{\pi}}{4 N} \int_{0}^{\infty} d \sigma \sigma^{-3 / 2} \tilde{\Phi}_{1}(\sigma)=\frac{1}{2 N} \int_{0}^{\infty} d \vec{x}^{2}\left(\vec{x}^{2}\right)^{1 / 2} \Phi_{1}\left(\vec{x}^{2}\right)
$$

We notice that the function $\Phi_{2}\left(x^{2}\right)$ does not contribute to $c_{0}$ and the large $b^{2}$ behavior of the Wilson loop is governed entirely by the function $\Phi_{1}\left(x^{2}\right)$.

Summarizing, we conclude that the Wilson loop has different behavior at small and large impact vectors $b^{2}$ :

$$
\ln W_{0}\left(b^{2}, Q^{2}\right)=-\ln \frac{Q^{2}}{Q_{0}^{2}} c_{1} b^{2}, \quad \text { for } b^{2} \ll \lambda^{2}
$$

and

$$
\ln W_{0}\left(b^{2}, Q^{2}\right)=-\ln \frac{Q^{2}}{Q_{0}^{2}} c_{0} \sqrt{b^{2}}, \quad \text { for } b^{2} \gg \lambda^{2}
$$

where $\lambda$ is the characteristic length defined by the properties of the nonlocal correlator (5.8). The nonperturbative scales $c_{0}$ and $c_{1}$ are given by (6.12) and (6.4), respectively. We recall that the expressions (5.18), (6.13) and (6.14) were obtained by solving the evolution equation (5.15) and therefore they are valid up to corrections independent on $Q^{2}$. In the expressions (1.5) and (1.6) the later corrections are parameterized by the scale $g_{1}$.

\subsection{Ansatz for the nonlocal correlator}

Calculating the scales $c_{1}$ and $c_{0}$ we shall rely on particular nonperturbative ansatz for the nonlocal correlator (5.8). Since their values depend on the behavior of the nonlocal correlator (5.8) at large euclidian distances $x^{2}<0$, we may apply the results of lattice calculations of this correlator.

According to [19, 14, the nonlocal correlator (5.8) is exponentially decreasing function of the space separation $\vec{x}^{2}$ which can be parameterized at large distances as

$$
\Phi_{1}\left(\vec{x}^{2}\right) \sim \Phi \mathrm{e}^{-\frac{|\vec{x}|}{\lambda}}, \quad \frac{d \Phi_{2}}{d x^{2}}\left(\vec{x}^{2}\right) \sim \Phi^{\prime} \mathrm{e}^{-\frac{|\vec{x}|}{\lambda}}
$$


with $\lambda$ being the correlation length, $\Phi$ and $\Phi^{\prime}$ being the overall normalization scales. This ansatz leads to the following expression for the function $\tilde{\Phi}(\sigma)$ defined in (5.8)

$$
\tilde{\Phi}_{1}(\sigma)=\frac{\Phi}{\sqrt{4 \pi \lambda^{2}}} \sigma^{-3 / 2} \exp \left(-\frac{1}{4 \sigma \lambda^{2}}\right)
$$

and to similar expression for the function $\tilde{\Phi}_{2}(\sigma)$. The lattice determination of these scales gives [19]

$$
\Phi=7.2 \times 10^{8} \Lambda_{L}^{4}, \quad \lambda=1 /\left(183 \Lambda_{L}\right)
$$

with the lattice QCD parameter

$$
\Lambda_{L}=(0.005 \pm 0.0015) \mathrm{GeV}
$$

In addition, $\left|\Phi^{\prime}\right| \approx \Phi / 10$ and therefore we may neglect the contribution of the $\Phi_{2}$-function to (5.18). Then, substituting the ansatz (6.15) into the definition (6.10) and (6.12) we get the following expressions for the coefficients

$$
c_{1}=\frac{\Phi \lambda^{2}}{2 N}=3584 \Lambda_{L}^{2}, \quad c_{0}=\frac{2 \Phi \lambda^{3}}{N}=78 \Lambda_{L}
$$

Their values depend strongly on the lattice scale $\Lambda_{L}$. Choosing this scale at the upper boundary $\Lambda_{L}=0.0065 \mathrm{GeV}$, or equivalently $\lambda=0.17 \mathrm{fm}$, and using (6.17) we estimate the scales $(6.19)$ as

$$
c_{1}^{(\text {latt })}=0.15 \mathrm{GeV}^{2}, \quad c_{0}^{(\text {latt })}=0.51 \mathrm{GeV} .
$$

Let us compare our predictions with the phenomenological parameterization Eqs. (1.5)(1.7). To this end we rewrite (1.5) in equivalent form keeping the $\ln Q$-dependent part only

$$
\ln W_{0}^{(\text {phen })}\left(b^{2}, Q^{2}\right)=-S_{\text {nonPT }}=-\ln \frac{Q^{2}}{Q_{0}^{2}} \frac{g_{2}}{2} b^{2} .
$$

We observe that this phenomenological expression matches (6.13) at small $b^{2}$ as

$$
c_{1}^{\text {(phen) }}=\frac{g_{2}}{2}=0.17_{-0.04}^{+0.04}(\mathrm{GeV})^{2} .
$$

This expression is in agreement with our calculation (6.20).

It follows from our analysis that the parameterization (1.6) and (6.21) of nonperturbative corrections to the Sudakov form factor is correct only for small $b^{2} \ll \lambda^{2}$, or equivalently for the transverse momenta $q^{2} \gg \lambda^{-2}$. We would like to stress that although the numerical expressions (6.17) are sensitive to the particular ansatz for the nonlocal correlator (5.8), the general form of the small $b^{2}$ expansion (6.2) is uniquely fixed. According to (6.14), we expect that at large $b^{2}$ (or small transverse momenta $\left.q^{2} \ll \lambda^{-2}\right)$ the asymptotic behaviour of the Wilson loop, and as a consequence nonperturbative correction to the Sudakov form factor, should have different form (6.14) with the scale $c_{0}$ given by $(6.12)$ and $(6.17)$. 


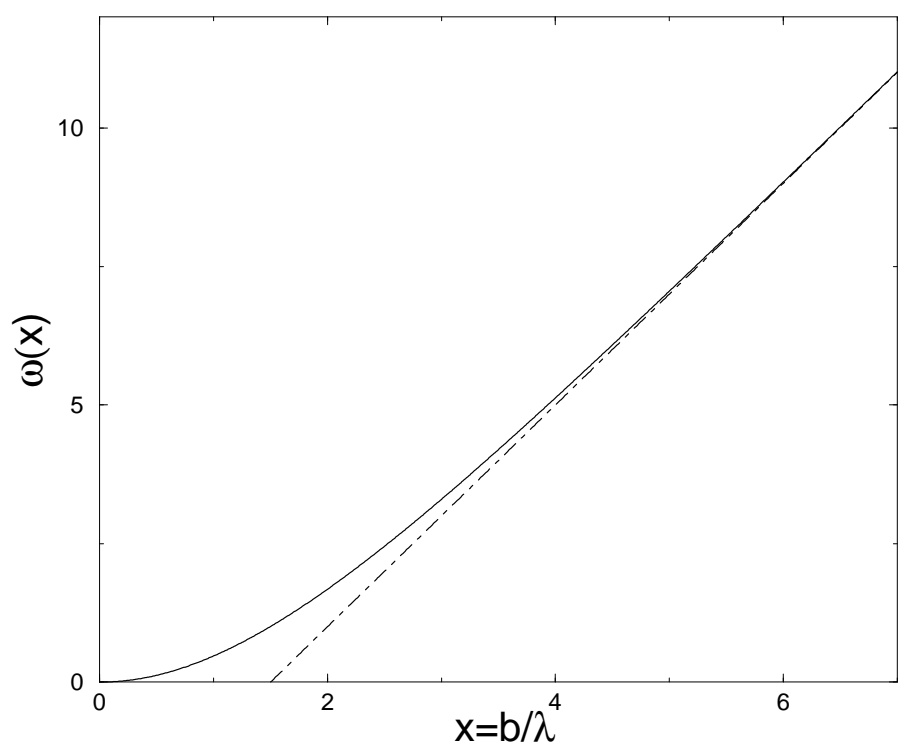

Figure 2: The profile function $w(b / \lambda)$ governing the $b$-dependence of nonperturbative contribution to the Sudakov form factor (6.23). The dash-dotted line describes the asymptotic behaviour (6.14).

To summarize, the nonperturbative corrections to small momentum distribution of lepton pairs in the Drell-Yan process are described by the expression (5.18) which is valid up to $Q$-independent corrections. The asymptotic behavior of the exponent of (5.18) at large and small impact vectors is given by (6.13) and (6.14), respectively. Combining together (5.18) with nonperturbative ansatz for the nonlocal gauge field correlator, (6.15), one can find the expression for the nonperturbative factor that is valid for an arbitrary $b^{2}$. Another advantage of the ansatz (6.16) that integration in (5.18) can be performed explicitly leading to the following expression for the nonperturbative factor

$$
\ln W_{0}\left(b^{2}, Q^{2}\right)=-\ln \frac{Q^{2}}{Q_{0}^{2}} \frac{\Phi \lambda^{4}}{N} w(b / \lambda),
$$

with the profile function

$$
w(x)=\mathrm{e}^{-x}(3+x)-3+2 x
$$

which is plotted in Fig. 2. Going over to the transverse momentum space we define nonperturbative weight function $f_{\text {nonPT }}\left(k^{2}, Q^{2}\right)$ as

$$
f_{\text {nonPT }}\left(k^{2}, Q^{2}\right)=\int \frac{d^{2} b}{(2 \pi)^{2}} \mathrm{e}^{i \vec{b} \cdot \vec{k}} W_{0}\left(b^{2}, Q^{2}\right)
$$

Then, inserting the factor $W_{0}\left(b^{2}, Q^{2}\right)$ into the integrand of (1.1) we find that nonperturbative effects produce a smearing of the perturbative $q$-distribution, Eq. (1.1), 
with the weight function $f_{\text {nonPT }}\left(k^{2}, Q^{2}\right)$. Substituting (5.18) into (6.25) and taking into account (6.15) we obtain the $k$-dependence of the weight function $f_{\text {nonPT }}\left(k^{2}, Q^{2}\right)$ as shown in Fig. 3.

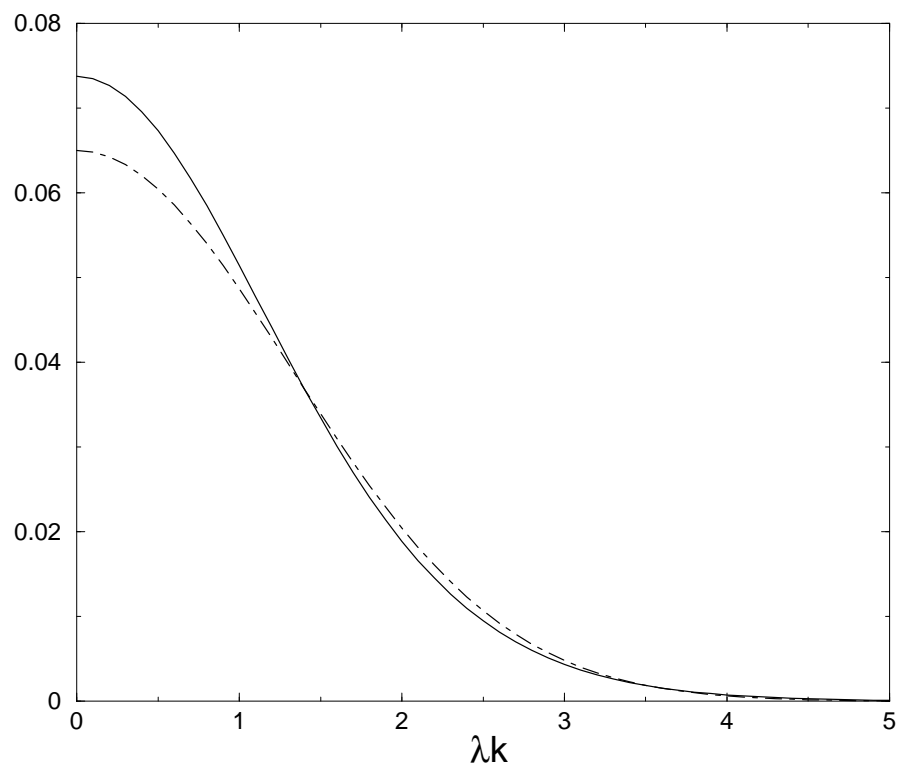

Figure 3: The nonperturbative primordial distribution $f_{\text {nonPT }}\left(k^{2}, Q^{2}\right)$ at $Q=91 \mathrm{GeV}$ and $Q_{0}=1.6 \mathrm{GeV}$. The dash-dotted line describes the Gaussian distribution corresponding to (6.13).

\section{Conclusion}

In this paper we have studied nonperturbative corrections to the transverse momentum distribution of vector bosons in the Drell-Yan process. Factorizing out the Sudakov effects due to soft gluons we were able to express their contribution to the distribution in the form of the vacuum averaged Wilson loop operator. We have demonstrated that the Wilson loop take into account both perturbative and nonperturbative corrections. The former are resummed through the evolution equation whereas the later define the boundary conditions for their solutions. Analyzing large order perturbative corrections to the Sudakov form factor we found that perturbation theory provides an ambiguous contribution to power corrections $\sim\left(b^{2} \Lambda^{2}\right)^{n}$ associated with the IR renormalons. The IR renormalon contribution suggests the general structure of nonperturbative corrections and it can be absorbed into nonperturbative boundary conditions for the Wilson loop.

We calculated the nonperturbative contribution to the Sudakov form factor using the expansion of the Wilson loop over vacuum fields supplemented with the expression for nonlocal gauge invariant field strength correlator. Although the Wilson loop is defined in an essentially Minkowski kinematics, the part of the nonperturbative 
contribution depending on the invariant mass of the produced vector boson $Q^{2}$ is governed by asymptotics of the correlator at large space-like (Euclidean) separations and therefore can be calculated using conventional nonperturbative methods. Applying the results of lattice calculations we found that the obtained expression for the nonperturbative power corrections is in qualitative agreement with known phenomenological expressions at large transverse momenta and deviate from them at small transverse momenta.

\section{Acknowledgements}

I am most grateful to G. Korchemsky for numerous stimulating discussions and suggestions. I would also like to thank T. Binoth, J.-Ph. Guillet and E. Pilon for useful conversations and G. Sterman for critical reading of the manuscript.

This work was supported in part by the EU network 'Training and Mobility of Researchers', contract FMRX-CT98-0194.

\section{References}

[1] Yu.L. Dokshitzer, D.I. D’Yakonov, and S.I. Troyan, Phys. Lett. 79B (1978) 269;

G. Parisi and R. Petronzio, Nucl. Phys. B154 (1979) 427;

J. Collins and D. Soper, Nucl. Phys. B193 (1981) 381; B213 (1983) 545(E);

G. Altarelli, R.K. Ellis, M. Greco, and G. Martinelli, Nucl. Phys. B246 (1984) 12;

C.T.H. Davies and W.J. Stirling, Nucl. Phys. B244 (1984) 337.

[2] C.T.H. Davies, B.R. Webber, and W.J. Stirling, Nucl. Phys. B256 (1985) 413;

P.B. Arnold and R.P. Kauffman, Nucl. Phys. B349 (1991) 381.

[3] J.C. Collins, D.E. Soper, and G. Sterman, Nucl. Phys. B250 (1985)199.

[4] G. Ladinsky and C.-P. Yuan, Phys. Rev. D 50 (1994) 4239;

F. Landry, R. Brock, G. Ladinsky, and C.P. Yuan, Phys. Rev. D63 (2001) 013004, hep-ph/9905391.

[5] R.K. Ellis and S. Veseli, Nucl. Phys. B511 (1998) 649, hep-ph/9706526;

S. Frixione, P. Nason, and G. Ridolfi, Nucl. Phys. B542 (1999) 311, hep-ph/9809367;

A. Kulesza and W.J. Stirling, Nucl. Phys. B555(1999) 279, hep-ph/9902234, JHEP 0001 (2000) 016, hep-ph/9909271.

[6] G.P. Korchemsky and G. Sterman, Nucl. Phys. B437 (1995) 415, hep-ph/9411211.

[7] A. Guffanti and G. E. Smye, JHEP 0010 (2000) 025 hep-ph/0007190;

E. Laenen, G. Sterman and W. Vogelsang, hep-ph/0010080;

J.W. Qiu and X. Zhang, hep-ph/0012058, hep-ph/0012348;

A.I. Karanikas and N.G. Stefanis, hep-ph/0101031. 
[8] G.P. Korchemsky, Phys. Lett. 217B (1989) 330, 220B (1989) 629.

[9] A. Bassetto, M. Ciafaloni and G. Marchesini, Phys. Rept. 100 (1983) 201.

[10] R. Meng, F. I. Olness and D. E. Soper, Phys. Rev. D54 (1996) 1919;

P. M. Nadolsky, D. R. Stump and C. P. Yuan, Phys. Rev. D61 (2000) 014003 hepph/0012261.

[11] G.P.Korchemsky and A.V.Radyushkin, Nucl. Phys. B283 (1987) 342; Phys. Lett. 279B (1992) 359.

[12] G.P.Korchemsky, Mod. Phys. Lett. A4 (1989) 1257; I.A.Korchemskaya and G.P.Korchemsky, Phys. Lett. 287B (1992) 169.

[13] M. Beneke, Phys. Rept. 317 (1999) 1.

[14] H. G. Dosch, V. I. Shevchenko and Y. A. Simonov, hep-ph/0007223;

Y. A. Simonov, Phys. Usp. 39 (1996) 313 hep-ph/9709344;

H. G. Dosch, Prog. Part. Nucl. Phys. 33 (1994) 121; and references therein.

[15] M.A.Shifman, Nucl. Phys. B173 (1980) 13.

[16] S.V. Mikhailov and A.V. Radyushkin, Sov. J. Nucl. Phys. 49 (1989) 494; 52 (1990) 697.

[17] G.Sterman, in Perturbative Quantum Chromodynamics, Ed. D.W.Duke and J.F.Owens, AIP Conference Proceedings, No.74, American Institute of Physics, New York, 1981;

J.G.M.Gatheral, Phys. Lett. 133B (1983) 90;

J.Frenkel and J.C.Taylor, Nucl. Phys. B246 (1984) 231.

[18] G.P. Korchemsky and G. Sterman, unpublished.

[19] A. Di Giacomo and H. Panagopoulos, Phys. Lett. B285 (1992) 133. 